\title{
Philonsorbonne
}

11 | 2017

Année 2016-2017

\section{Vertu éthique et rationalité pratique chez Aristote. Note sur la notion d'hexis proairetikê}

Pierre-Marie MOREL

\section{(2) OpenEdition}

Journals

Édition électronique

URL : https://journals.openedition.org/philonsorbonne/892

DOI : $10.4000 /$ philonsorbonne.892

ISSN : $2270-7336$

Éditeur

Publications de la Sorbonne

\section{Édition imprimée}

Date de publication : 1 janvier 2017

Pagination : 141-153

ISSN : 1255-183X

\section{Référence électronique}

Pierre-Marie MOREL, « Vertu éthique et rationalité pratique chez Aristote. Note sur la notion d'hexis proairetikê », Philonsorbonne [En ligne], 11 | 2017, mis en ligne le 06 janvier 2017, consulté le 08 juin 2021. URL : http://journals.openedition.org/philonsorbonne/892 ; DOI : https://doi.org/10.4000/ philonsorbonne.892 


\title{
Vertu éthique et rationalité pratique chez Aristote. Note sur la notion d'hexis proairetikê
}

\author{
Pierre-Marie MOREL \\ (Université Paris 1 Panthéon-Sorbonne - UMR 7219 GRAMATA)
}

Lorsqu'il définit la vertu du caractère ou vertu éthique (courage, générosité, tempérance, etc.) au livre II de l'Éthique à Nicomaque, Aristote commence par montrer à quel genre elle appartient, en éliminant les genres concurrents. Elle n'est ni une affection ou passion $(\pi \alpha \dot{\theta} \theta \varsigma)$, ni une faculté

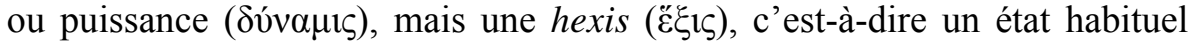
qui constitue à la fois une disposition acquise et, comme nous le verrons, une inclination ${ }^{1}$. La vertu éthique sera donc une hexis permettant d'agir de manière courageuse, généreuse, ou encore tempérante. Pour spécifier ensuite le type d'hexis auquel appartient la vertu éthique ${ }^{2}$, il précise qu'il s'agit

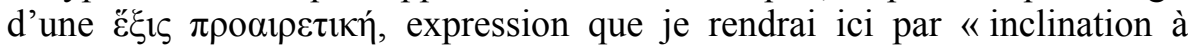
décider » :

« La vertu est donc une inclination à décider, située dans un juste milieu relatif à nous fixé par la raison et comme le fixerait le prudent. C'est en outre un juste milieu entre deux vices, l'un par excès et l'autre par défaut, et cela parce que les uns sont en défaut et les autres en excès par rapport à ce qui doit

1. «Après cela, il faut examiner ce qu'est la vertu. Puisque donc il y a trois types d'états psychiques, les affections, les facultés et les inclinations, la vertu sera l'un d'eux. (...) Si donc les vertus ne sont ni des affections ni des facultés, elles ne pourront être que des inclinations.

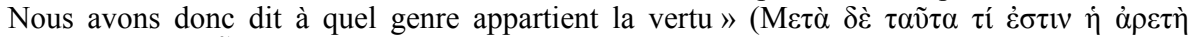

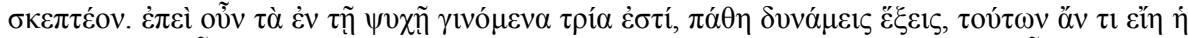

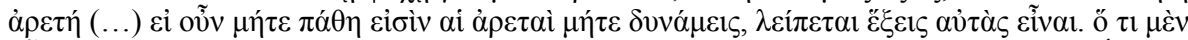

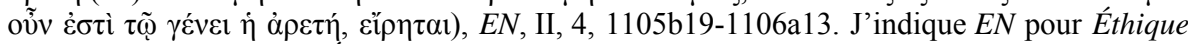
à Nicomaque et EE pour Éthique à Eudème.

2. Je ne suis pas entièrement sur ce point, P. Aubenque, La Prudence chez Aristote, Paris, P.U.F, 1963, p. 119-120, pour qui l'adjectif spécifie la vertu éthique en l'opposant à la vertu naturelle, parce que la première atteste notre responsabilité. Je conviens tout à fait que la présence de la proairesis dans la définition de la vertu éthique souligne le caractère responsable et volontaire de cette dernière, mais l'opposition à la vertu naturelle n'est pas explicite dans ce contexte précis. 
être, dans le domaine des affections et dans celui des actions, alors que la vertu trouve et choisit le milieu. C'est pourquoi, selon la substance, c'est-à-dire selon l'énonciation de ce qui fait son essence même, la vertu est un juste milieu, bien que selon le meilleur et le convenable, ce soit un sommet $»^{3}$.

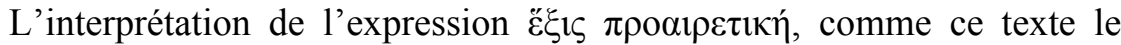
laisse déjà penser, recouvre des enjeux fondamentaux pour la compréhension de l'éthique aristotélicienne. La formule se donne incontestablement pour une définition de la vertu éthique, comme l'indique clairement la précision « selon la substance, c'est-à-dire selon l'énonciation de ce qui fait son

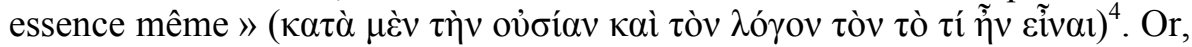
telle qu'elle se présente ici, cette définition relie directement la vertu éthique, par le biais de l'invocation du phronimos, à une vertu intellectuelle ou dianoétique, la prudence ou sagesse pratique ( $\varphi \rho o ́ v \eta \sigma r \varsigma)$, qui, comme on le sait, représente l'excellence en matière de délibération. L'expression $\tilde{\varepsilon}^{\prime} \xi 1 \zeta$

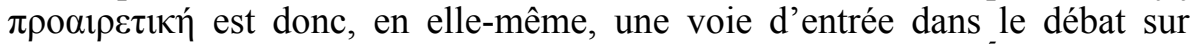
l'intellectualisme moral supposé de l'éthique aristotélicienne ${ }^{5}$ ou, en des termes moins abstraits, sur la part que prennent les opérations proprement intellectuelles - qu'elles relèvent de la science ou du calcul pratique - dans les actions moralement significatives. Si la vertu éthique implique par elle-même la proairesis, voire la phronêsis, cela signifie qu'elle est déjà intellectuelle en quelque manière, et qu'elle n'est pas intégralement assimilable à une disposition non rationnelle ou à un pur sentiment moral. Je voudrais montrer dans ce qui suit que cette expression, prise dans son contexte d'énonciation, invite en réalité à dépasser l'opposition entre une interprétation strictement intellectualiste de la psychologie morale d'Aristote et la lecture alternative que, par commodité, j'appellerai « sentimentaliste ».

Partons toutefois du clivage exégétique. La formule reflète en effet les raisons objectives de l'opposition entre la lecture intellectualiste de l'éthique d'Aristote et les interprétations qui soulignent à l'inverse sa dimension intuitive, sentimentale ou empirique. Selon ces dernières, la vertu éthique, parce qu'elle oriente le désir de manière irréfléchie et spontanée - et non

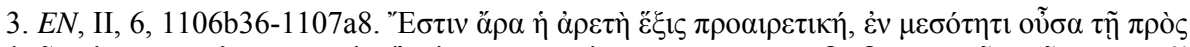

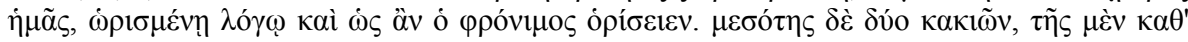

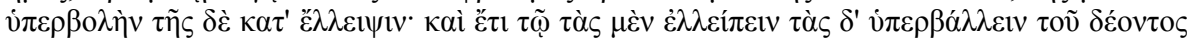

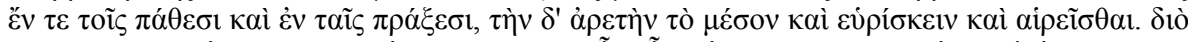

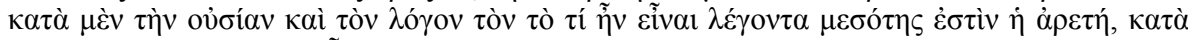

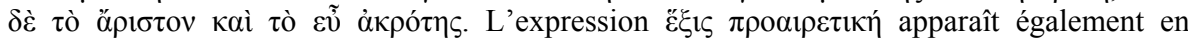
$E N$, VI, 2, 1139a22-23; EE, II, 10, 1227b5-11.

4. Aspasius, dans son commentaire à l'Éthique à Nicomaque, estime qu'Aristote livre là une définition en bonne et due forme, en accord avec les recommandations formulées dans les Seconds analytiques : " il est clair qu'il a donné < dans ce passage> la définition correcte

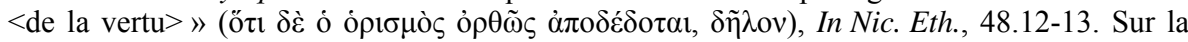
conformité aux Seconds analytiques, voir 48.31-49.2.
}

5. Question sur laquelle, pour une mise au point récente, je renvoie à L. Monteils-Laeng, Agir sans vouloir. Le problème de l'intellectualisme moral dans la philosophie ancienne, Paris, Classiques Garnier, 2014. 
par un calcul dépendant de la partie rationnelle de l'âme - vers une fin bonne, serait une sorte de refuge du sentiment, la part proprement émotionnelle de la détermination morale ${ }^{6}$. La prudence, vertu intellectuelle par laquelle nous délibérons sur les moyens en vue d'atteindre les fins droites, bien qu'elle aussi se définisse par opposition à une rationalité plus accomplie - et s'oppose ainsi à la fois à la science et à la sophia, sagesse proprement théorique -, serait, somme toute, le versant le plus intellectuel de la doctrine aristotélicienne de l'action ${ }^{7}$. La distinction entre vertu éthique et prudence est généralement reconnue comme allant de soi et elle est bien attestée par les textes. En premier lieu, elle se fonde sur les éléments de psychologie morale que nous donnent les traités éthiques, et qui distribuent les types de vertus selon les facultés de l'âme :

« $<$ la faculté $>$ qui possède la raison sera double également : il y aura d'un côté ce qui la possède éminemment et en soi-même, de l'autre ce qui la possède en obéissant, comme on obéit à son père. Or la vertu elle aussi se définit selon cette différence. Nous disons en effet que certaines sont des vertus intellectuelles et les autres du caractère. La sagesse théorique, la capacité de compréhension et la prudence sont des vertus intellectuelles, tandis que la libéralité et la tempérance sont des vertus du caractère. En parlant en effet du caractère de quelqu'un, nous ne disons pas qu'il est sage ou capable de comprendre, mais qu'il est doux ou tempérant. Toutefois nous louons aussi le sage pour son inclination, et les inclinations qui appellent nos louanges, nous les appelons vertus $»^{8}$.

\begin{abstract}
6. Voir en ce sens D.S. Hutchinson, «Ethics» dans J. Barnes (éd.), The Cambridge Companion to Aristotle, Cambridge, Cambridge University Press, 1995, p. 195-232, p. 213 : «The moral virtues are settled habits of character which express themselves in the correct emotional response ». Dans le même sens, L.A. KosmAn, « Being Properly Affected: Virtues and Feelings in Aristotle's Ethics ", dans A.O. Rorty (éd.), Essays on Aristotle's Ethics, Berkeley-Los Angeles-London, University of California Press, 1980, p. 103-116, note que les vertus éthiques n'ont pas pour seule finalité de réaliser des actions, mais qu'elles sont également des manières de réguler nos émotions, et qu'en ce sens elles sont directement en rapport avec les pathê. De fait, lorsque l'Éthique à Eudème formule la définition de la vertu

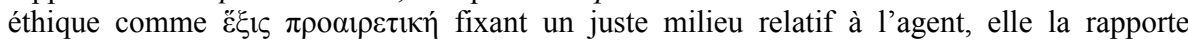
directement aux affections de plaisir et de peine (EE, II, 10, 1227b5-11).
\end{abstract}

7. Même si, comme l'a souligné la lecture fameuse de P. AuBENQue, op. cit., par exemple p. 49-51, la prudence elle-même traduit la bipolarité de l'éthique aristotélicienne, entre la recherche d'une norme universelle de l'excellence et l'estimation empirique des conditions concrètes de l'action humaine.

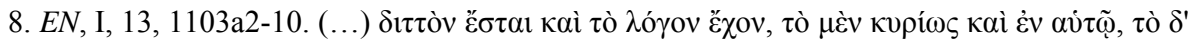

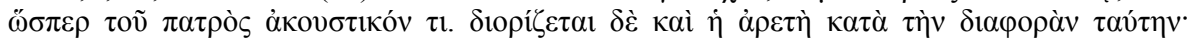

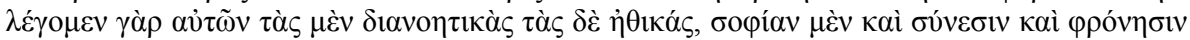

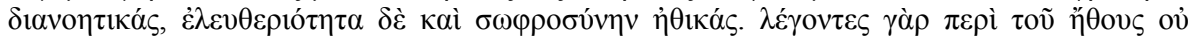

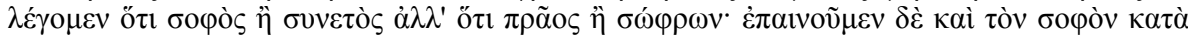

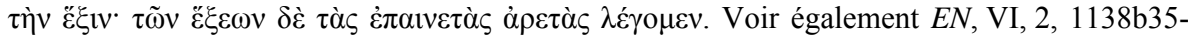
1139a3 : «En divisant les vertus de l'âme, nous avons dit que les unes se rapportent au caractère, les autres à la raison. Puisque nous avons traité des vertus du caractère, parlons du

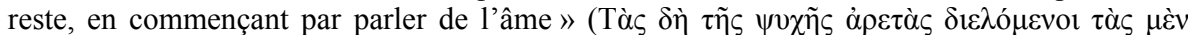

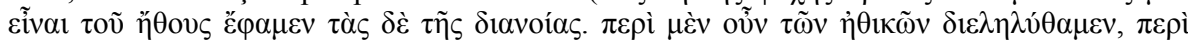

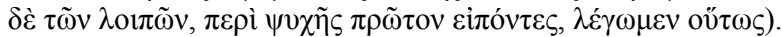


L'ouverture du livre II de l'Éthique à Nicomaque, de plus, distingue clairement les vertus éthiques des vertus intellectuelles (y compris donc la phronêsis), à la fois par référence aux facultés de l'âme et en fonction de leurs modes d'acquisition respectifs :

«Il est établi que la vertu est double, vertu intellectuelle et vertu du caractère ; c'est principalement à l'instruction que la vertu intellectuelle doit sa genèse et son développement, et c'est précisément pourquoi elle a besoin d'expérience et de temps, tandis que la vertu du caractère résulte de l'habitude, d'où lui est également venu son nom, qui est une légère déclinaison de "habitude" »".

Aristote maintient d'ailleurs cette distinction, $\mathrm{y}$ compris là où il entend montrer que vertus éthiques et phronêsis sont indissociables : les premières garantissent la rectitude de la fin poursuivie, tandis que celle-ci assure celle des actions intermédiaires, ou moyens, en vue de cette fin ${ }^{10}$.

On ne peut qu'être frappé, toutefois, par l'irruption du phronimos dans la définition de EN, II, 6 , alors que la phronêsis, tout au moins dans la séquence que constituent les livres I et II de l'Éthique à Nicomaque, n'a pas encore été définie ${ }^{11}$, ni confrontée à la vertu éthique. Le juste milieu que constitue la vertu éthique est en effet déterminé par la raison, et cela « comme le fixerait» (ou le «déterminerait») «l'homme prudent ${ }^{12}$. Il

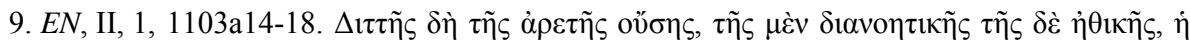

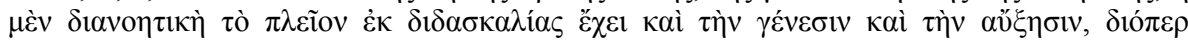

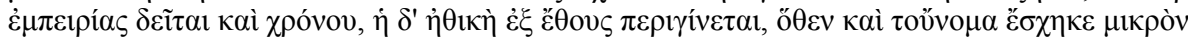

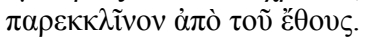

10. EN, VI, 13, 1144a6-9; 1145a5-6 ; EE, II, 11, 1227b22-25; 39.

11. Je n'entends pas ici me prononcer sur l'ordre des livres de l'Éthique à Nicomaque, ni des livres qui lui sont propres par rapport aux livres qui figurent également dans l'Éthique à Eudème, pas plus que sur la chronologie respective des deux traités. Je ne veux donc pas fonder l'analyse sur l'antériorité supposée du livre II de l'Éthique à Nicomaque par rapport au livre VI, qui est le deuxième des livres communs. Il me semble en tout cas, d'une part, que le livre II prend la suite du livre I et qu'ils font partie d'une séquence commune, et, d'autre part, que l'analyse des vertus éthiques trouve naturellement place dans les premières phases de l'analyse - avant donc les développements sur la prudence que l'on trouve au livre VI. Il y a en effet parallélisme de structure entre les deux premiers livres de l'Éthique à Nicomaque et les deux premiers livres de l'Éthique à Eudème. Dans l'Éthique à Nicomaque, l'analyse de la vertu éthique vient au livre II, après l'exposé des principes de l'enquête sur le bien proprement humain (livre I dans l'Éthique à Nicomaque), tout comme, dans l'Éthique à Eudème, à partir de II, 2. Notons, enfin, que le passage parallèle de l'Éthique à Eudème,

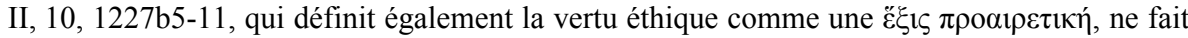
pas mention du phronimos. Le problème que l'on pose ici concerne donc spécifiquement l'Éthique à Nicomaque.

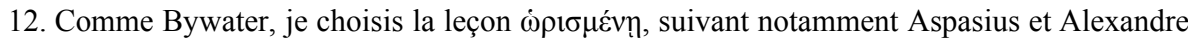
d'Aphrodise, considérant qu'il est plus naturel de parler de définition ou de détermination à propos du milieu, qu'à propos de la vertu elle-même (ce qui doit être le cas si l'on accepte la

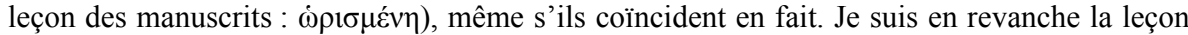
manuscrite (cette fois à la différence de Bywater), en lisant ẃs plutôt que õ̃ («comme le déterminerait...»). 
n'est pas très simple de savoir quelle valeur Aristote veut donner au

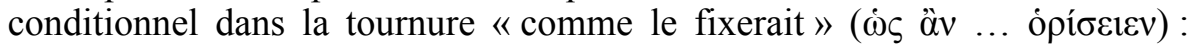
s'agit-il d'un irréel, qui impliquerait que le milieu visé par la vertu éthique lui est propre, qu'il ne relève donc pas de la prudence, mais qu'il est tel que la prudence le fixerait si une telle tâche lui incombait ? S'agit-il, à l'inverse, d'un potentiel - comme la grammaire, du reste, le voudrait -, indiquant que le prudent lui-même ne procèdera pas autrement pour fixer la norme de la conduite? Dans ce cas, vertu éthique et prudence seraient non seulement étroitement liées, parce que l'une ne va pas sans l'autre, mais plus ou moins identifiables l'une à l'autre en fait. Il est du reste significatif que la prudence, par l'intermédiaire du phronimos, soit mentionnée dans une phrase ayant pour objet, comme on l'a vu, la définition même de la vertu éthique, ce qui suggère que le lien avec la phronêsis entre dans son essence. La distinction entre vertu éthique et vertu intellectuelle ou dianoétique tend dès lors à s'estomper.

H. Lorenz ${ }^{13}$ a insisté à juste titre sur cette particularité, en réaction aux interprétations «sentimentalistes », notamment celle déjà mentionnée de D. S. Hutchinson. Lorenz s'oppose à l'idée selon laquelle les vertus du caractère seraient des aptitudes habituelles à éprouver de manière appropriée des sentiments tels que le plaisir, le désarroi, la colère ou la honte et qu'elles relèveraient exclusivement de la partie irrationnelle de l'âme (p. 177-178). Il note par exemple que la justice, qui est une vertu de ce type, réside pour une part importante dans la capacité qu'a la personne juste de saisir des raisons appropriées permettant d'agir d'une manière donnée (p. 178). De fait, et plus généralement, la proairesis, décision ou choix ${ }^{14}$, loin d'être une opération strictement rationnelle, ou à l'inverse un pur élan de désir, est présentée par Aristote comme un désir raisonnant ou un intellect désirant ${ }^{15}$. La proairesis est à la fois le résultat ultime de la bouleusis, opération intellectuelle de délibération sur les moyens en vue d'une action bonne, et l'ultime impulsion mentale qui précède l'action elle-même. C'est donc une opération commune à la partie rationnelle de l'âme et à la partie désirante, partie qu'Aristote qualifie d'irrationnelle, même si elle peut se soumettre aux injonctions de la raison, à la différence de la partie irrationnelle de premier niveau, dévolue aux fonctions végétatives et nutritives.

13. Voir H. Lorenz, "Virtue of Character in Aristotle's Nicomachean Ethics », Oxford Studies in Ancient Philosophy XXXVII, 2009, p. 177-212.

14. Ou encore « intention », comme le préconisent P. Aubenque, op. cit., p. 119 sq., ou encore A. Merker, Une Morale pour les mortels. L'éthique de Platon et d'Aristote, Paris, Les Belles Lettres, 2011, en comprenant le terme en son sens premier de «tension vers » un objet. Je considère pour ma part que le terme " intention », même s'il présente l'avantage de soustraire la proairesis au paradigme strictement intellectualiste de l'arbitrage abstrait, introduit une ambiguïté. Il peut en effet laisser croire qu'elle se situe encore au niveau des velléités d'actions, par opposition à l'action effective, alors que l'action doit être effectivement réalisée, pour Aristote, si l'on veut qualifier moralement l'agent et sa conduite.

15. Voir EN, III, 5, 1113a11; VI, 2, 1139b4-5. 
Par retour, la vertu éthique, parce qu'elle implique la proairesis, participera directement de la rectitude de la décision ${ }^{16}$. Les vertus éthiques, ainsi comprises, seraient donc, selon H. Lorenz, de véritables «états rationnels » (《rational states »), parce qu'elles sont en partie constituées par le fait de bien délibérer (p. 195). Lorenz franchit un pas supplémentaire en considérant que « la disposition qui permet à l'individu vertueux d'adopter des fins bonnes et la phronêsis se combinent de manière à constituer un état dispositionnel unifié ${ }^{17}$, en sorte que la vertu éthique est en elle-même «partiellement constituée par la prudence ${ }^{18}$.

Pour compléter cette approche, j'observe que, d'un strict point de vue

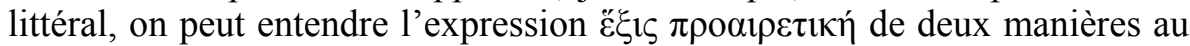
moins, selon que l'on fait porter l'accent sur la notion d'hexis ou sur l'adjectif proairetikos. Dans le premier cas, elle invite à souligner l'aspect dispositionnel et habituel de la vertu, mais aussi sa dimension irrationnelle, s'il est vrai que la vertu ainsi définie ne va pas sans désir et qu'elle suppose une tendance vers le bien; elle ne découle donc pas, ou pas exclusivement, d'une attitude intellectuelle. Si l'on insiste plutôt sur le fait que la vertu éthique est ici liée à la proairesis, on met alors l'accent sur l'idée de conduite rationnelle, la vertu orientant le choix, en rendant l'agent capable d'exercer une activité rationnelle déterminant l'action. Compte tenu du contexte du passage - où il s'agit, encore une fois, d'énoncer la différence spécifique de la vertu éthique parmi les autres hexeis -, il me paraît clair que l'accent porte sur la dimension « proairétique». Aristote ne cherche pas ici à limiter le poids de la vertu éthique en indiquant que, par rapport à la décision effective, elle n'est encore qu'une potentialité de décision; il l'investit au contraire d'une responsabilité écrasante dans la réalisation de l'action morale, en précisant que ce qui fait son essence, et ce qui par conséquent lui confère sa fonction propre, c'est une certaine détermination, déjà rationnelle, du juste milieu entre des vices opposés.

Faut-il pour autant, en versant la pièce au dossier déjà signalé, l'invoquer au bénéfice exclusif de la position intellectualiste, contre son opposée ? J'opterai pour d'une troisième lecture, assez proche par ailleurs de celle de H. Lorenz. Il me semble en fait qu'Aristote veut suggérer ici que les deux dimensions des états qui permettent la conduite morale, aspect dispositionnel et dimension intellectuelle, sont tous deux originaires. L'hexis vertueuse, bien qu'elle ne soit pas assimilable en tant que telle à une opération de calcul et de délibération, n'est pas exempte pour autant de toute

16. H. Lorenz, op. cit., p. 179 : «makes decision correct».

17. H. Lorenz, op. cit., p. 200 : «the virtuous person's disposition to adopt good goals and phronesis do combine to constitute a unified dispositional state ».

18. "partly constituted by phronesis », H. Lorenz, op. cit., p. 200. De même, L. Brown, «Why Is Aristotle's Virtue of Character a Mean? Taking Aristotle at His Word (NE ii 6)», dans R. Polansky (éd.), The Cambridge Companion to Aristotle's Nicomachean Ethics, Cambridge, Cambridge University Press, 2014, p. 64-80, va jusqu'à considérer que la détermination de la mesotês dans la vertu est «le rôle de la raison, précisément du phronimos » (p. 70). 
composante rationnelle parce qu'elle participe par essence de la rationalité

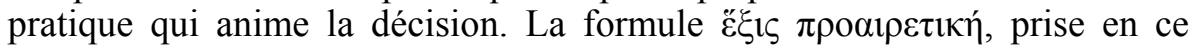
sens, serait une façon, particulièrement condensée et synthétique, de traduire la double nature, à la fois intellectuelle et dispositionnelle, de la vertu du caractère.

La question qui demeure - et c'est sur ce point que je m'écarterai en partie de la lecture de Lorenz - est de savoir comment comprendre la distinction entre vertu éthique et proairesis, distinction qu'Aristote, de fait, ne remet pas en cause. L'objet de la proairesis est en effet ce qui a été « prédélibéré » ( $\pi \rho \circ \beta \varepsilon \beta o v \lambda \varepsilon v \mu \varepsilon ́ v o v)$ et la décision «s'accompagne de raison et de

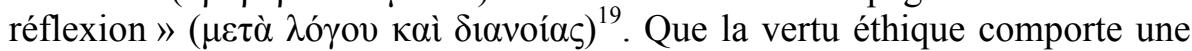
part de raison, nous le constaterons plus loin; il n'en demeure pas moins qu'Aristote, à ma connaissance, ne lui attribue pas la réflexion proprement dite. En quel sens peut-on dire dès lors que la vertu éthique participe déjà de la rationalité pratique, alors qu'elle semble constituer l'élément le plus «sentimental» de la psychologie morale d'Aristote? Faut-il considérer, selon la position que Lorenz me parait choisir, qu'il y a dans la vertu éthique elle-même, outre la composante irrationnelle, une composante rationnelle et effectivement délibérative, voire qu'elle est déjà décision ? Ou bien doit-on plutôt dire que la vertu éthique n'est pas en elle-même décisionnelle, mais qu'elle est rationnelle en un autre sens ?

Ces difficultés apparaissent d'ailleurs dès que l'on tente de préciser

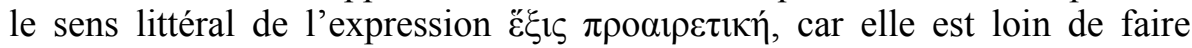
consensus, comme l'atteste la diversité des traductions proposées. Le sens de la formule varie assez nettement, selon qu'on estime que l'hexis est déjà et en elle-même liée à la décision ${ }^{20}$, ou bien qu'elle lui est antérieure et la préconditionne $^{21}$; ou encore selon qu'on y voit une simple aptitude ${ }^{22}$ ou bien

19. EN, III, 4, 1112a15-16. Il n'est pas nécessaire de trancher ici le débat sur le sens du préfixe $\pi \rho o-$ dans le terme $\pi \rho 0 \alpha i ́ \rho \varepsilon \sigma i \varsigma$, qui peut être compris comme un indicateur temporel (d'antériorité), ou bien comme un indicateur logique (de disjonction) ; dans le second cas, on déciderait non pas «avant» mais «de préférence à». Voir en ce sens J.-B. Gourinat, «Délibération et choix dans l'éthique aristotélicienne», dans G. Romeyer Dherbey et G. Aubry (éd.), L'Excellence de la vie. Sur l'Éthique à Nicomaque et l'Éthique à Eudème d'Aristote, Paris, Vrin, 2002, p. 95-124.

20. Voir, par exemple, R. Bodeüs, Aristote. Éthique à Nicomaque, trad. fr., intr. et notes, Paris, GF-Flammarion, 2004 : "état décisionnel »; M. Pakaluk, Aristotle's Nicomachean Ethics. An Introduction, Cambridge, Cambridge University Press, 2005, p. 105 : «a state involving deliberate purpose $»$.

21. J.-Y. Jolif, dans R.-A. Gauthier, J.-Y. Jolif, , Aristote. L'Éthique à Nicomaque, Intr., trad. et commentaire, Louvain, 1970: "un état habituel qui dirige la décision »; C. Rowe dans C. Rowe, S. Broadie, Aristotle. Nicomachean Ethics, Oxford, Oxford University Press, 2002 : « a disposition issuing in decisions ».

22. C. Natali, La Saggezza di Aristotele, Napoli, Bibliopolis, 1989, p. 76 : «abitudine a scegliere »; J. Tricot, Aristote. Éthique à Nicomaque, trad. fr. et notes, Paris, Vrin, 1967 : " disposition à agir d'une façon délibérée » (en EN, II, 6), "disposition capable de choix » (en $E N, \mathrm{VI}, 2$ ). 
une tendance et un facteur productif ${ }^{23}$. Parfois, le traducteur choisit l'option de l'imprécision, espérant peut-être se protéger ainsi par la neutralité ${ }^{24}$. Ces divergences confirment en tout cas que nous sommes face à deux questions : (a) l'hexis dont il est question est-elle plutôt une disposition, une capacité ou état, ou bien faut-il y voir une tendance, une inclination à agir de telle ou telle manière ? (b) la vertu du caractère est-elle déterminée, en quelque sorte de l'extérieur, par la rationalité pratique contenue dans la composante « proairétique », ou bien est-elle déjà rationnelle?

Concernant la première question (a), on peut comprendre hexis selon une acception passive (état) ou selon une acception forte et dynamique, qui conçoit l'hexis non seulement comme une aptitude acquise mais encore comme une tendance ou inclination. Considérée dans son processus d'acquisition ou du point de vue génétique, comme effet d'une habitude résultant de la répétition d'actes semblables, l'hexis peut être caractérisée de manière satisfaisante comme une simple disposition ou aptitude. En son sens générique, elle n'implique pas nécessairement une tendance ou un désir et correspond mieux à ce que nous entendons par «disposition». Ainsi, la vertu intellectuelle qu'est la science est une $\check{\varepsilon} \xi 1 \varsigma \dot{\alpha} \tau$ ' $\delta \varepsilon \iota \kappa \tau \iota \kappa \eta^{25}$, une « disposition à démontrer ». Comprise toutefois comme un état du caractère, et par conséquent de notre manière habituelle de désirer, elle détermine l'agent à désirer telle action plutôt que telle autre. Ainsi comprise, l'hexis en question est plutôt une tendance ou une inclination. Dans tous les cas, elle a statut, non pas de pure puissance, mais de réalisation première, déjà déterminée positivement par la fin à laquelle elle tend, comme la disposition savante du savant par l'exercice effectif de la science.

La mention du juste milieu dans la définition de la vertu éthique a en effet une connotation dynamique, qui tient au pouvoir littéralement « stochastique» de cette dernière :

« $\mathrm{Si}$, en outre, la vertu est plus exacte et meilleure que tout art, comme l'est aussi la nature, elle visera le milieu. Je veux parler de la vertu du caractère, car c'est elle qui porte sur les affections et les actions, en lesquelles précisément il y a excès, défaut et milieu $»^{26}$.

23. C. Natali, Aristotele. Etica Nicomachea, Roma-Bari, 1999: «stato abituale che produce scelte $»$.

24. D. Ross, Aristotle, Nicomachean Ethics, dans J. Barnes, The Complete Works of Aristotle. The Complete Oxford Translation, vol. II, Bollingen Series LXXI 2, Princeton, Princeton University Press, (1984) 1991 : « state concerned with choice».

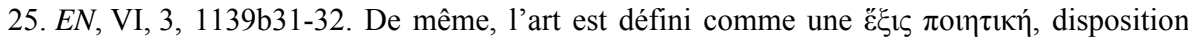
à produire, en $E N, \mathrm{VI}, 4,1140 \mathrm{a} 20-21$.

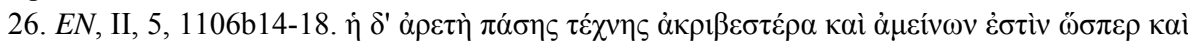

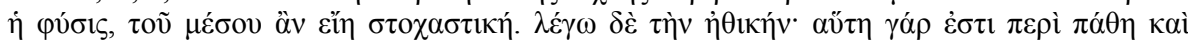

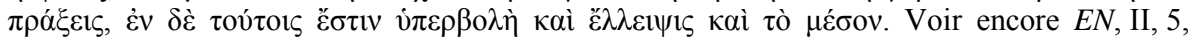
1106b27-28 : «La vertu sera donc une forme de juste milieu, au sens où elle consiste dans

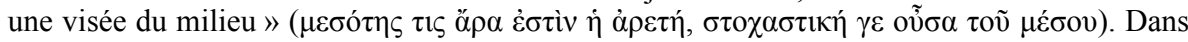
le même sens, voir les arguments proposés par A. Merker, Le Principe de l'action humaine selon Démosthène et Aristote. Hairesis - Prohairesis, Paris, Les Belles Lettres, 2016, p. 343-346, 
La vertu n'est donc pas uniquement une capacité personnelle intermédiaire, la qualité morale d'un caractère sachant se tenir dans les limites de la juste mesure entre les vices opposées; elle est encore une « visée ", et en ce sens une tendance, en direction du juste milieu objectif que réalisera l'action bonne ${ }^{27}$.

La traduction par « inclination $»^{28}$ présente en outre cet avantage qu'elle rend mieux compte de la dynamique interne de l'action morale : celle-ci résulte en effet d'une orientation positive du souhait ou désir raisonnable (boulêsis) de l'agent en direction du bien. La vertu éthique est donc, par ellemême, une certaine orientation du désir vers le bien, qui résulte de l'habitude d'accomplir des actes d'une nature déterminée.

Se pose enfin la question (b) de savoir si la proairesis est l'objet et le but externe de la vertu éthique, comme plusieurs traductions le donnent à penser, ou bien si, comme Lorenz le soutient, elle lui est inhérente. Aux arguments déjà avancés en faveur de cette dernière interprétation, j'ajoute que l'inclination morale vise (et souhaite) la fin, qui est l'action bonne, et non pas la décision elle-même, qui n'est pas la fin ultime. On comprendra donc que la vertu est une inclination "décisionnelle» au sens où elle participe du processus de décision en vue de l'action. Raison et désir sont de ce point de vue inextricablement liés ${ }^{29}$. L'inclination à décider doit donc, en principe, être déjà porteuse de décision.

Ajoutons que, plus fondamentalement, l'imbrication de la vertu éthique et de la décision prudente est conforme à la manière dont Aristote décrit l'expérience morale. Celle-ci n'est pas la résultante d'une coopération de facultés et d'opérations initialement hétérogènes les unes aux autres, comme si la composante désirante et la composante rationnelle, la visée de la fin et l'estimation des moyens adéquats, étaient discernables dans l'analyse de la conduite et comme si chacune opérait pour son œuvre propre. Le courage en acte, ce n'est pas seulement l'actualisation de la disposition courageuse ; c'est aussi être courageux de la manière qui convient, au moment approprié,

notamment, p. 346 : « La vertu comme hexis prohairetikê prédispose à une visée complète du but, avec la capacité de l'atteindre effectivement».

27. Sur la distinction, et l'articulation, entre l'état de juste milieu ( $\mu \varepsilon \sigma o ́ \tau \eta \varsigma)$ qui constitue

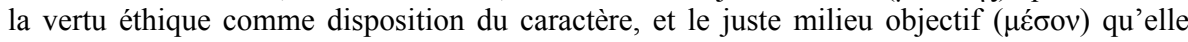
tend à réaliser, voir L. Brown, art. cit.

28. Sur les problèmes que pose l'articulation, chez Aristote et dans la tradition aristotélicienne postérieure, de l'inclination et de la décision, je renvoie à P.-M. Morel, «Inclination et décision. Le problème de l'assentiment chez Aristote et Alexandre d'Aphrodise ", dans L.-Jaffro (éd.), Croit-on comme on veut? Histoire d'une controverse, Paris, 2013, p. 27-45. Je rejoins C. Murgier, Éthiques en dialogue. Aristote lecteur de Platon, Paris, Vrin, coll. "Textes et traditions », 2013, qui relie directement l'hexis à l'agir et insiste sur sa dimension d'inclination en indiquant que «la vertu éthique est (...) une habitude, mais habitude d'agir» (p. 140) et, plus loin, que l'habitude impliquée par la vertu éthique, " est moins une structure statique qu'une tendance qui nous porte à l'action » (p. 141).

29. Voir en ce sens L. Monteils-LAENG, op. cit., p. 275-285, à propos de ce qu'elle appelle «l'implication de la prohairèsis dans la définition de l'hexis èthikè » (p. 284). 
avec les gens qui conviennent, par rapport aux objets qui le justifient; or tout cela implique précisément la prudence dans la décision. Le texte fameux où Aristote affirme que la prudence d'un côté et les vertus éthiques de l'autre sont interdépendantes confirme d'ailleurs que toutes les vertus, bien qu'elles ne soient pas des «sciences » comme le voulait Socrate, supposent la raison:

«La vertu n'est pas seulement l'inclination qui est conforme à la raison droite, mais celle qui implique la présence de la raison droite ; or la prudence est en la matière raison droite. Ainsi, alors que Socrate pensait que les vertus étaient des raisons (car pour lui elles sont toutes des sciences), pour notre part nous considérons qu'elles impliquent la présence de la raison. Il est donc clair, en vertu de ce que nous avons dit, qu'il est impossible d'être véritablement homme de bien sans prudence, ni prudent sans la vertu du caractère $» 30$.

Cela ne doit pas pour autant nous conduire à abolir toute distinction entre vertu éthique et prudence, et cela pour au moins quatre raisons, que je résumerai ainsi :

(i) La prudence est une vertu intellectuelle, ce qui la distingue à plusieurs titres des vertus éthiques, ainsi que nous l'avons vu.

(ii) La vertu du caractère est une puissance déjà orientée vers son actualisation et vers le contraire positif - elle n'est pas indifféremment puissance du bien ou du mal, mais puissance du bien -, avec statut de réalisation première, parce qu'elle est une potentialité déterminée et non pas une potentialité neutre, ou pure dunamis, d'accomplir A plutôt que non-A. En ce sens, la disposition ou inclination morale ne peut pas ne pas viser la fin qui lui correspond. Aristote le dit très clairement au début du livre $\mathrm{V}$ de l'Éthique à Nicomaque, en rappelant ce principe jamais démenti qu'il n'y a qu'une seule puissance et une seule science des contraires, tandis qu'une hexis produit un effet déterminé et ne peut pas produire l'effet contraire. La santé ne produit pas ce qui est contraire à la santé ${ }^{31}$. Or la sagesse pratique suppose une aptitude radicale à choisir entre deux partis contraires, $\mathrm{A}$ ou non-A, et c'est ce qui nous permet de dire que l'agent est véritablement «principe et maître » de ses propres actions ${ }^{32}$. Dès lors, si la vertu éthique est rationnelle, comme le veut son caractère « proairétique », ce ne peut être

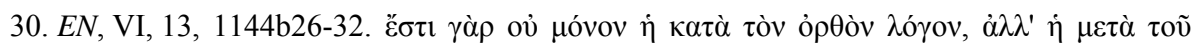

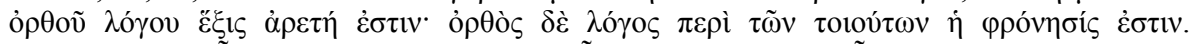

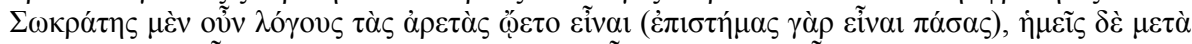

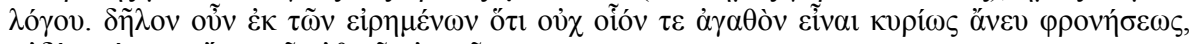

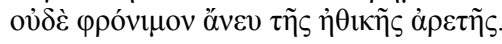

31. EN, V, 1, 1129a11-16. Ce texte pose indirectement le problème de savoir si l'on peut réellement changer de caractère ou de dispositions. Il est tout au moins clair que, si l'on agit contrairement à notre disposition, ce n'est pas en vertu de cette disposition elle-même, mais du fait d'une autre disposition ou d'une attitude intempérante à l'égard de la règle.

32. Voir EE, II, 6, 1223a4-9. 
au même titre que la vertu qui s'exerce, comme bonne délibération, dans le choix d'un parti à prendre ${ }^{33}$.

(iii) La vertu du caractère vise le milieu, ce qui n'est pas le cas de la prudence, parce que les vertus intellectuelles réalisent leur excellence absolument et non pas dans une échelle de degrés. Comme le dit habilement Aspasius, « la vertu intellectuelle n'est pas un milieu, car il ne s'agit pas de savoir de manière moyenne, et sans excès, mais il sera bien mieux de savoir autant qu'il est possible $\gg{ }^{34}$.

(iv) Les vertus éthiques visent les fins ; la prudence estime les moyens.

Il faut donc supposer que, s'il est vrai que la vertu éthique est déjà rationnelle, elle l'est en un autre sens, en quelque sorte basique, par rapport à la rationalité qui est à l'œuvre dans la délibération sur les moyens - et c'est sur ce point précis que je m'écarte de l'analyse de H. Lorenz. En quoi cette saisie rationnelle non discursive peut-elle consister? Elle peut prendre quatre aspects.

(a) La vertu, tout d'abord, sera rationnelle autant qu'un habitus peut l'être, c'est-à-dire non pas comme un raisonnement en acte (opération qui, $\mathrm{du}$ reste, revient aux vertus intellectuelles), mais plutôt comme capacité à raisonner, en vue du bien. Fixant la fin et le milieu, elle n'est pas encore le calcul pratique effectif, qui de toute façon ne saurait s'effectuer qu'en situation, mais elle est ce qui va orienter celui-ci vers la fin bonne. La vertu éthique est donc présente dans la délibération comme règle générale d'action, comme celle qui sert de majeure dans les syllogismes pratiques ${ }^{35}$.

(b) Les états du caractère, en tant qu'ils relèvent du désir supérieur, témoignent de la capacité qu'a celui-ci d'obéir à la raison, de sorte que, bien qu'ils ne soient pas « rationnels » au sens pleinement actif et prescriptif d'un raisonnement pratique, ils sont au moins "raisonnables", ainsi que nous l'avons vu dans le texte de $E N, \mathrm{I}, 13,1103 \mathrm{a} 2-10$. On peut d'ailleurs estimer que l'éducation morale - notamment si elle dépend de la science du législateur - et les actions délibérées antérieures de l'agent, en œuvrant à l'acquisition d'habitudes vertueuses, préconditionnent par des voies rationnelles et délibérées des dispositions qui ne sont pas en elles-mêmes délibératives.

33. Comme le dit très justement C.D.C. Reeve, Aristotle on Practical Wisdom. Nicomachean Ethics VI, Cambridge (MA) - London, Harvard University Press, 2013, p. 94 : « Like the crafts and sciences, and unlike the potentialities we possess by nature, the virtues both involve reason (NE II 6 1106b36-1107a2) and are acquired by frequently or habitually doing the relevant activities (...). But unlike other potentialities that involve reason, and like those possessed by nature, the virtues are potentialities not for contraries but for a single thing ».

34. Aspasius, In Nic. Eth., 47.19-21.

35. Sur ce qu'il serait plus exact d'appeler « les syllogismes du faisable » (oi $\sigma v \lambda \lambda o \gamma 1 \sigma \mu$ ò̀ $\tau \tilde{\omega} v$ $\pi \rho \alpha \kappa \tau \tilde{\omega} v)$, et sans entrer dans les difficultés que posent non seulement l'expression, mais encore le propos d'Aristote quand il en use, voir notamment $E N$, VI, 13, 1144a31-32 ; VII, 5, $1147 \mathrm{a} 28$ sq. ; Mouvement des animaux, 7, 701a12-13. 
(c) Parce qu'elle se situe dans une proportion géométrique entre deux vices, proportion déterminée par la situation et les propriétés de l'agent c'est un juste milieu « relatif à nous $»^{36}-$, la vertu éthique vise un milieu exprimable en termes de rapport mathématique. C'est en ce sens que ce milieu est « fixé par la raison comme le fixerait le prudent ». Entre l'absence de rationalité qui caractérise l'affect pur de plaisir ou de peine et la rationalité discursive de la délibération, il y a donc place pour la saisie spontanément rationnelle, parce que d'emblée proportionnelle, du milieu ${ }^{37}$. Il

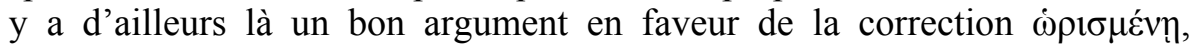
généralement acceptée par les traducteurs contre la leçon $\dot{\rho} \iota \mu \varepsilon^{\prime} \eta \eta$ : c'est le milieu qui est « déterminé » par la raison, comme appréciation spontanée du rapport impliqué par le désir vertueux.

(d) Comme l'indique clairement le dernier texte cité $^{38}$, la vertu éthique est, non pas la prudence elle-même, mais une disposition conforme à la raison droite et unie à elle. En ce sens, «elle implique la présence de la raison » (meta logou), ce qui, une fois encore, n'en fait pas un raisonnement à proprement parler. La prudence, à l'inverse, est elle-même la raison droite (VI, 13, 1144b27-28).

En résumé, le logos qui dirige la vertu de l'intérieur, et qui fixe le milieu, n'est pas un logismos, un raisonnement du type de ceux que produisent les vertus intellectuelles. Il est donc distinct du calcul délibératif dont la prudence est la vertu. La rationalité immanente à la vertu éthique est à la fois la règle générale d'action et le rapport, au sens mathématique du terme, qu'implique le moyen terme.

Le point le plus délicat reste sans doute la présence du phronimos dans la définition de la vertu éthique. La tournure « comme le fixerait le prudent » signifie peut-être que la vertu atteint le milieu avec la même efficacité et la même précision que le prudent le fait, ainsi qu'on peut le constater dans l'expérience courante. De fait, il ne peut s'agir que du même milieu, un milieu géométrique, parce que « relatif à nous », entre deux vices opposés. La vertu éthique le vise toutefois de manière spontanée, non calculée, et en indiquant la fin, tandis que la prudence a pour tâche propre d'administrer par le raisonnement pratique les moyens permettant de parvenir à cette fin. Invoquer la conduite du prudent, comme on mentionne une attitude exemplaire, pour illustrer la façon dont la vertu éthique vise le juste milieu,

36. Voir EN, II, 5, 1106a26-b28. L'exemple fameux du régime diététique de l'athlète, qui diffère de celui du sportif occasionnel, illustre bien la nécessaire adaptation de la juste mesure à la situation et aux capacités propres de l'agent ; le juste milieu ne sera pas à égale distance des extrêmes, comme s'il s'agissait d'une proportion arithmétique, mais dans un rapport de proportion géométrique intégrant le paramètre de l'agent.

37. J'estime, en accord avec C. Rapp, "What Use Is Aristotle's Doctrine of the Mean?», dans B. Reis (éd.), The Virtuous Life in Greek Ethics, Cambridge, Cambridge University Press, 2006, p. 99-126, que la visée du juste milieu par la vertu ne relève pas d'une procédure de décision, ni du reste d'une procédure mathématique.

38. EN, VI, 13, 1144b26-32. 
ne signifie donc pas que vertu éthique et prudence composent un même état dispositionnel.

L'insistance sur la dimension décisionnelle de l'inclination morale conduit donc à concevoir cette dernière comme un principe déjà rationnel, non pas toutefois au sens où elle serait en elle-même une opération de calcul des moyens, mais parce qu'elle oriente la décision vers le bien en vertu d'un rapport, et par conséquent d'une certaine forme de logos. Ce qui peut passer pour un paradoxe traduit en fait l'unité et la cohérence de la théorie aristotélicienne de l'action morale, au-delà du clivage traditionnel entre la lecture intellectualiste et ses opposées.

Dans cette double manière de participer de la rationalité, la théorie aristotélicienne de la vertu puise une part significative de son originalité : il y a dans la pratique une manière d'agir rationnellement qui implique, non seulement une procédure intellectuelle de calcul des moyens (ainsi qu'une dimension empirique et intuitive nécessaire à la saisie des conditions singulières de l'action et à la prudence), mais aussi une forme basique de

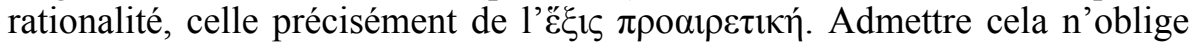
pas à nier la fonction, indiscutable, du désir dans la réalisation de l'action. Le type d'hexis qu'est la vertu éthique est en effet une tendance (et non pas une simple aptitude) et la proairesis elle-même est un certain type de désir. Aristote, en affirmant que le désir est, dans sa forme la plus complexe, « raisonnable », interdit en fait que l'on oppose, dans l'analyse des conditions de l'action, une composante purement rationnelle à une autre, qui ne le serait absolument pas. Ce n'est pas en raisonnant que l'on atteint le juste milieu, mais ce n'est pas non plus sans une certaine forme de raison ${ }^{39}$.

39. Je tiens à remercier les participants du colloque de Covilhã, en novembre 2015, en particulier José Manuel Santos et Laurent Jaffro, pour la très stimulante discussion que nous avons eue sur ces questions. 